\title{
EXPERIMENTO CON BIOL DE SUBPRODUCTOS DE AZÚCAR PARA MAYOR RENDIMIENTO ECOLÓGICO EN EL CULTIVO DE LECHUGA (LACTUCA SATIVA L.)*
}

\author{
Edwin Gálvez Torres ${ }^{*}$ \\ Universidad Nacional Faustino Sánchez Carrión \\ egalvez@unjfsc.edu.pe \\ José Legua Cárdenas ${ }^{* *}$ \\ Universidad Nacional Faustino Sánchez Carrión \\ jlegua@unjfsc.edu.pe \\ Dante Daniel Cruz Nieto ${ }^{* * * *}$ \\ Universidad Nacional Faustino Sánchez Carrión \\ daniel2262@hotmail.com \\ Félix Caro Soto ${ }^{* * * * *}$ \\ Universidad Nacional Faustino Sánchez Carrión \\ fcaro@unjfsc.edu.pe
}

* Los autores agradecen al Gobierno Nacional que a través de FOCAM (Fondo de Desarrollo Socioeconómico de Camisea) proporciona a la UNJFSC financiamiento para la realización de proyectos de investigación como el presente trabajo.

** Edwin Gálvez Torres es ingeniero químico egresado de la Universidad Nacional de Trujillo. Actualmente es docente universitario en la UNJFSC. Doctorado en Ciencias Ambientales por la UNJFSC.

*** José Legua Cárdenas es ingeniero químico egresado de la Universidad Nacional Mayor de San Marcos. Actualmente es autoridad universitaria en la UNJFSC. Doctorado en Ingeniería por la UNFV.

**** Dante Daniel Cruz Nieto es ingeniero agrónomo egresado de la Universidad Nacional Santiago Antúnez de Mayolo. Tiene estudios de maestría en Gestión y Ecología Ambiental por la UNJFSC.

${ }^{* * * * *}$ Félix Caro Soto es licenciado en Administración, egresado de la UNJFSC. Actualmente es docente universitario en la UNJFSC. Doctorado en Administración por la UNJFSC. 
Fecha de recepción: agosto de 2019 Fecha de aceptación: diciembre de 2019

ReSUMEN: La presente investigación versa en torno al aprovechamiento de residuos derivados de la caña de azúcar, para reducir la contaminación en el suelo. El objetivo es determinar la concentración ( $\mathrm{ml}$ de biol/l de agua) adecuada de biol, para el mayor rendimiento de lechuga. El fertilizante se obtuvo de la composición de $16.5 \mathrm{~kg}$ de rastrojo, $16.5 \mathrm{~kg}$ de guano de cuy, $11 \mathrm{~kg}$ de bagazo y 111 de vinaza.

Se empleó el modelo estadístico del diseño de bloques complemente al azar que consta de 5 tratamientos que incluye el testigo y la dosis estándar: $\mathrm{T}_{1}$ con $0.0, \mathrm{~T}_{2}$ con $250, \mathrm{~T}_{3}$ con $500, \mathrm{~T}_{4}$ con $750, \mathrm{~T}_{5}$ con $1000 \mathrm{ml} \mathrm{de}$ biol sobre 2001 de agua. Se evaluó en campo y laboratorio. Con los datos obtenidos se operaron con el análisis de varianza y Duncan.

Los resultados determinaron que el $\mathrm{T}_{5}$ con $12.02 \mathrm{tn} / \mathrm{ha}$ de rendimiento, en longitud de planta con $26.92 \mathrm{~cm}$, con un peso por lechuga $165.83 \mathrm{~g}$, y un diámetro ecuatorial de $24.80 \mathrm{~cm}$. No obstante, el $\mathrm{T}_{4}$ obtuvo mayor longitud de raíz con $9.00 \mathrm{~cm}$. En análisis foliar, el $\mathrm{T}_{5}$ con $1000 \mathrm{ml}$ de biol obtuvo baja concentración de los elementos (N, P, K y otros), sin embargo, esta cantidad influyó en rendimiento de lechuga.

Palabras clave: Biol, dosis, residuos de la caña de azúcar y rendimiento. 


\section{EXPERIMENT WITH BIOL OF SUGARCANE BYPRODUCTS FOR GREATER ECOLOGICAL PERFORMANCE IN LETTUCE CULTIVATION (LACTUCA SATIVA L.)}

AвSTRACT: This research deals with the use of residues derived from sugar cane, to reduce contamination in the soil. The objective is to determine the appropriate concentration ( $\mathrm{ml}$ of biol / 1 of water) of biol, for the highest yield of lettuce. The fertilizer was obtained from the composition of $16.5 \mathrm{~kg}$ of stubble, $16.5 \mathrm{~kg}$ of guano guinea pig, $11 \mathrm{~kg}$ of bagasse and $11 \mathrm{l}$ of stillage. The completely randomized block design statistical model consisting of 5 treatments including the control and the standard dose was used: T1 with 0.0, T2 with 250, T3 with 500, T4 with 750, T5 with 1000 $\mathrm{ml}$ of biol over $200 \mathrm{l}$ of water. It was evaluated in the field and laboratory. The obtained data were operated with the analysis of variance and Duncan.

The results determined that the T5 with $12.02 \mathrm{tn} /$ ha of yield, in plant length with $26.92 \mathrm{~cm}$, with a weight per lettuce $165.83 \mathrm{~g}$, and an equatorial diameter of $24.80 \mathrm{~cm}$. However, T4 obtained greater root length with $9.00 \mathrm{~cm}$. In foliar analysis, T5 with $1000 \mathrm{ml}$ of biol obtained a low concentration of the elements (N, P, K and others), however, this amount influenced lettuce yield.

KeYwords: Biol, Dose, Sugarcane waste and Yield. 


\section{EDWIN GÁLVEZ TORRES, JOSÉ LEGUA CÁRDENAS, DANTE DANIEL CRUZ NIETO Y FÉLIX CARO SOTO}

\section{Introducción}

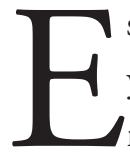

ste trabajo de investigación está basado en el desarrollo agrosostenible y ecológico, por lo que innova las labores convencionales, ya que reduce la aplicación de insumos químicos a la planta y al suelo. Para tal motivo se utilizó biol (fertilizante líquido) que se obtuvo a base de subproductos de la caña de azúcar (bagazo y vinaza) y otros materiales (estiércol de cuy y rastrojo de planta). Respecto a la dimensión agrosostenible, podemos corroborar las afirmaciones dadas por la Organización de las Naciones Unidas para la Alimentación y la Agricultura (2002), según las cuales el abono orgánico a menudo crea la base para el uso exitoso de los fertilizantes minerales. La combinación de abono orgánico / materia orgánica y fertilizantes minerales ofrece las condiciones ambientales ideales para el cultivo.

Con respecto al sostenimiento ecológico, donde la protección del suelo es importante en la actualidad, los nutrientes orgánicos cumplen con la función de proteger el sistema edáfico tal como manifiestan Bello, García y Díez (2004). De esa forma, es necesario un planteamiento nuevo en la investigación y gestión de la materia orgánica y, sobre todo, en las investigaciones sobre compostaje. Estas deben dar énfasis en las interrelaciones existentes entre compost-suelo-cultivo, centrándose en una investigación multidisciplinaria sobre ecobiología del compost que tenga en cuenta las características agroecológicas del cultivo y especialmente la ecología y biología del suelo.

La investigación propuso la utilización del biol producido mediante el bagazo y vinaza de la caña de azúcar como alternativa orgánica a la producción de lechuga y con ello minimizar la contaminación ambiental mediante la quema de estos desechos. Lo afirmado coincide con Bustamante 
(2014) respecto a la contaminación del aire, que señala que esta industria genera a través de emisiones de partículas como hollín y humo debido a la utilización de combustóleo y bagazo procedentes de la quema y requema de la caña de azúcar.

La caña de azúcar, desde sus inicios, ha tenido como derivados principales al azúcar, el alcohol y el papel. En estas últimas décadas se ha producido investigaciones donde se desarrollan fertilizantes líquidos como el denominado "Ajinofer" y otros no muy comercializados, por lo que su generosidad orgánica como nutriente que favorece a la planta y al suelo, en este caso es el tema de la presente investigación. Como manifiesta Ospina (2016), los compost maduros amortiguan los cambios de acidez en el suelo y forman compuestos estables, compost menos maduros continúan su transformación en el campo disminuyendo los contenidos de materia orgánica en el suelo.

El biol producido, a través de la presente investigación, ha favorecido la efectividad en los diferentes tratamientos demostrados en la altura de planta, diámetro ecuatorial y peso de la lechuga. Tal como manifestó el Fondo de Cooperación para el Desarrollo Social (2014), el biol estimula el crecimiento de las plantas y permite la protección contra las plagas y enfermedades. Además, ayuda a mantener el vigor de las plantas y soportar eventos extremos del clima. Es especialmente útil luego de heladas y granizada.

El biol ofrece a la planta una variedad de propiedades físicas y químicas, las cuales se manifiestan en la calidad y rendimiento de la planta. Como lo refirieron López, Andrade, Herrera, Gonzales y García (2015), el compost (base de subproductos de caña de azúcar) muestra indicadores positivos de calidad, cuantificándose la humedad de $59 \%$, el pH 8.2; la 
relación carbono-nitrógeno de 12.5; una densidad aparente de $0.55 \mathrm{~g} \mathrm{~cm}^{-3}$; el nitrógeno de $1 \%$; fósforo $1.3 \%$; potasio $1.1 \%$ y magnesio $1.1 \%$, así como una baja salinidad. Todo ello acarreó un resultado beneficioso para la producción ecológica.

\section{Materiales y Métodos}

$\mathrm{E}$ 1 presente trabajo de investigación toma como eje central las opciones concentraciones de biol a adicionar al cultivo de lechuga, según la referencia técnica y cientifica. La procedencia de las semillas de la lechuga utilizadas para la preparación de los almácigos es de la empresa Hortus. Asimismo, la locación escogida para llevar a cabo la investigación fué el Fundo Los Anitos, ubicado en la provincia de Barranca.

\subsection{Método de la investigación}

El experimento de diferentes concentraciones de biol aplicado al cultivo de lechuga tiene como objetivo obtener el mayor rendimiento ecológico, pues con esto se minimiza el consumo de fertilizantes químicos. Para llevarlo a cabo se probó en cinco tratamientos y se evaluó las características físicas y químicas. Con estas afirmaciones se establece que el presente es un método aplicado, pues se basa en obtener una concentración adecuada para mejorar el rendimiento. Tal afirmación proviene de López (2011), en tanto asegura que la investigación aplicada tiene como propósito de estudio la búsqueda de conocimiento con fines de aplicación inmediata a la realidad para modificarla o presentar solución a problemas prácticos en vez de formular teorías acerca de ellos. 


\subsection{Procedimiento del experimento}

Este experimento se realizó de la siguiente manera:

- Para la preparación del terreno se procedió a la respectiva la limpieza de campo, riego de machaco, oreo y empleo de maquinaria agrícola.

- Se tomó las respectivas muestras para el análisis de suelo, las mismas que proporcionaron un resultado (véase Tabla 1).

- Para la obtención del biol se procedió de la siguiente manera: el proceso de compostaje duró 112 días y su formulación fue de 16.5 $\mathrm{kg}$ de rastrojo, $16.5 \mathrm{~kg}$ de guano de cuy, $11 \mathrm{~kg}$ de bagazo y 11 litros de vinaza. Luego de obtener el compostaje se tomó $2 \mathrm{~kg}$ a la que se añadió 21 de agua, proporción 1:1 obteniéndose de esta manera el biol.

- Luego se procedió al trazo del terreno, para ubicar los tratamientos divididos en $\mathrm{T}_{1}$ con $0.0, \mathrm{~T}_{2} \operatorname{con} 250, \mathrm{~T}_{3} \operatorname{con} 500, \mathrm{~T}_{4} \operatorname{con} 750$ y $\mathrm{T}_{5}$ con $1000 \mathrm{ml}$ de Biol sobre $200 \mathrm{l}$ de agua.

- Se hizo el almácigo de lechuga que duró 37 días, seguidamente se trasplantó y después se aplicó el biol en dos momentos a los 7 y 14 días después del trasplante.

- Durante todo el proceso fenológico de la planta de lechuga se recogió, organizó, procesó y analizó los datos de altura, diámetro ecuatorial, peso y rendimiento comercial mediante los análisis estadísticos de ANVA y la Prueba de Duncan al $5 \%$ de error. 


\section{EDWIN GÁLVEZ TORRES, JOSÉ LEGUA CÁRDENAS, DANTE DANIEL CRUZ NIETO Y \\ FÉLIX CARO SOTO}

\subsection{Análisis de suelo}

La obtención de concentración de nutrientes del suelo se obtuvo mediante el análisis de $1 \mathrm{~kg}$ de muestra obtenida de forma aleatoria, siguiendo el marco conceptual de Quiroga y Bono (2002). Dichos autores mencionan que, para lograrlo, se requiere preparar una muestra compuesta de 10 a 15 submuestras extraídas de partes homogéneas del lote. A continuación, se muestra los resultados en cuadro Tabla 1 de análisis de suelo.

\section{Tabla 1}

\section{Análisis Básico de Fertilidad de Suelo, del Cultivo de Lechuga}

\begin{tabular}{|c|c|c|c|c|c|c|c|c|c|c|c|}
\hline \multirow{2}{*}{$\begin{array}{c}\text { C.E. } \\
\mathrm{mS} / \mathrm{cm} \\
1: 2.5\end{array}$} & \multirow{2}{*}{$\begin{array}{c}\mathrm{pH} \\
1: 2.5\end{array}$} & \multirow{2}{*}{$\begin{array}{c}\text { M.O. } \\
\%\end{array}$} & \multirow{2}{*}{$\begin{array}{l}\mathrm{N} \\
\%\end{array}$} & \multirow{2}{*}{$\begin{array}{c}\mathrm{P} \\
\mathrm{ppm}\end{array}$} & \multirow{2}{*}{$\begin{array}{c}\mathrm{K} \\
\mathrm{ppm}\end{array}$} & \multirow{2}{*}{$\begin{array}{c}\mathrm{CaCO}_{3} \\
\%\end{array}$} & \multicolumn{4}{|c|}{$\begin{array}{l}\text { Cationes intercambiables } \\
\text { Meq. } / 100 \text { g. suelo }\end{array}$} & \multirow[t]{2}{*}{ CIC-E } \\
\hline & & & & & & & $\mathrm{Ca}$ & $\mathrm{Mg}$ & $\mathrm{Na}$ & $\mathrm{k}$ & \\
\hline 1.19 & 6.88 & 1.37 & 0.07 & 12 & 212 & 1.76 & 16.99 & 0.68 & 0.29 & 0.54 & 18.50 \\
\hline
\end{tabular}

Adaptado de "Hoja de análisis de suelo. Código 105 -108," por Gálvez, Legua, Cruz \& Caro, 2018. Instituto Nacional de Investigación Agraria - Huaral.

Según los resultados del análisis de suelo, se muestra bajo porcentaje de N, media disponibilidad de Py K, baja concentración de materia orgánica, normal porcentaje de carbonatos, sin presencia de sales y $\mathrm{pH} 6.88$ neutro. Todo ello da a entender que este suelo es favorable para desarrollo agrícola y se debe incorporar materia orgánica (compost), para la siembra de lechuga. 
EXPERIMENTO CON BIOL DE SUBPRODUCTOS DE AZÚCAR PARA MAYOR RENDIMIENTO ECOLÓGICO EN EL CULTIVO DE LECHUGA (LACTUCA SATIVA L.)

Tabla 2

Concentraciones de Microelementos del Área Experimental

\section{Microelementos}

\begin{tabular}{lccc}
\hline Fe ppm & Zn ppm & Cu ppm & B ppm \\
103.90 & 7.62 & 11.04 & 1.56 \\
Normal & Bajo & Exceso & Bajo \\
\hline
\end{tabular}

Adaptado de "Hoja de análisis de suelo. Código 105 -108," por Gálvez, Legua, Cruz \& Caro, 2018. Instituto Nacional de Investigación Agraria - Huaral.

En cuanto a los microelementos, se determinó la baja concentración de $\mathrm{Zn}$ y Bo, y el aumento en $\mathrm{Fe}$ y $\mathrm{Cu}$, por lo que se recomendaría emplear compostaje para tener en condiciones normales los elementos de baja concentración. El motivo radica en que estos elementos influyen en el desarrollo de la planta y consecuentemente también favorecen en el rendimiento del cultivo.

\subsection{Aplicación estadística}

\subsubsection{Análisis de varianza}

Se utilizó al porcentaje de $1 \%$ y $5 \%$, para determinar si existe influencia de la dosis de compostaje en las variables de evaluación. Esto se basa con la afirmación de Núñez y Tusell (2007), quienes manifiestan que se presenta con frecuencia el problema de comparar el efecto que diversas circunstancias de tipo cualitativo ejercen sobre un cierto fenómeno. 
Con esta prueba de hipótesis se determinó qué tratamientos obtienen mayor cuantificación y, a la vez, si tiene relación homogénea. Esto se fundamenta con la expuesto por López y Gonzáles (2014), al manifestar que un procedimiento usado ampliamente para comparar todas las parejas de medias es el de la prueba de intervalos múltiples desarrollada por Duncan. Además exige, para ser exacto, que todos los tratamientos tengan el mismo número de repeticiones.

\subsubsection{Tratamiento del estudio}

La dosis de biol que se empleó en esta investigación, se observa en la Tabla 3. Asimismo, cabe afirmar que la concentración estándar es de 500 $\mathrm{ml}$ por $200 \mathrm{l}$ de agua. Dicha aplicación es corroborada por Cando y Malca (2015), al mencionar que en el cultivo hidropónico de lechuga (Lactuca sativa L.), el mejor rendimiento en peso se obtuvo con el tratamiento de $500 \mathrm{cc}$ de biol. 
Tabla 3

Aplicación de Dosis de biol, para los Tratamientos

\section{Dosis de aplicación de}

biol

Tratamiento

$\mathrm{ml}$ por 2001 de agua

$\mathbf{T}_{1}$

0.0

$\mathbf{T}_{2}$

250

$\mathbf{T}_{3}$

500

$\mathrm{T}_{4}$

750

$\mathrm{T}_{5}$

1000

\subsubsection{Análisis de biol}

Tabla 4

Análisis de Básico de Abono Orgánico (Biol)

\begin{tabular}{ccccccccccccc}
\hline $\mathrm{N}^{\circ}$ & & & C.E. & Humedad & $\mathrm{M} . \mathrm{O}$. & $\mathrm{C}$ & $\mathrm{N}$ & $\mathrm{P}_{2} \mathrm{O}_{2}$ & $\mathrm{~K}_{2} \mathrm{O}$ & $\mathrm{CaO}$ & $\mathrm{MgO}$ & $\mathrm{C} / \mathrm{N}$ \\
$\mathrm{Lab}$ & Muestra & $\mathrm{Ph}$ & $\begin{array}{c}\mathrm{mS} / \mathrm{cm} \\
\%\end{array}$ & $\%$ & $\%$ & $\%$ & $\%$ & $\%$ & $\%$ & $\%$ & \\
\hline 046 & Lixiviado & 7.75 & 4.00 & 99.53 & 36.95 & 21.43 & 1.60 & 0.90 & 0.48 & 4.98 & 0.20 & 13.39 \\
\hline
\end{tabular}

Adaptado de "Hoja de análisis de suelo. Código 105 -108," por Gálvez, Legua, Cruz \& Caro, 2018. Instituto Nacional de Investigación Agraria - Huaral.

La Tabla 4 muestra la baja concentración de nitrógeno, fósforo y potasio, sin embargo indica un buen porcentaje de materia orgánica, calcio y magnesio. También la relación carbono y nitrógeno es excesivo, lo cual muestra una fuente de energía. 


\section{EDWIN GÁLVEZ TORRES, JOSÉ LEGUA CÁRDENAS, DANTE DANIEL CRUZ NIETO Y FÉLIX CARO SOTO}

\section{Resultados y Discusión}

L a presente investigación tiene como objetivo obtener frutos ecológicos y sostenibles experimentando con diferentes concentraciones de biol. Este último se obtuvo a base de subproductos de la caña de azúcar como bagazo y vinaza, además de la mezcla con hierba seca y guano de cuy, de todo lo cual se obtuvo compostaje. De esta mezcla se tomó una muestra de $2 \mathrm{~kg}$ y se le añadió 2 l. de agua (proporción 1:1), y se dejó reposar anaeróbicamente por un espacio de tiempo de 1 mes. Luego filtro el producto obtenido y se preparó las diferentes concentraciones con el líquido filtrado y se aplicó al cultivo de lechuga, para luego evaluar los efectos del biol con el objetivo de confrontar sus resultados con el cultivo de control, puesto que la aplicación de estos compuestos influyen en el desarrollo de la planta. Tal aseveración es mencionada por Friedmann, Weil y Penner (2010), quienes exponen que de la producción de azúcar orgánica se pueden obtener subproductos para ser comercializados o reutilizados. El bagazo, bagacillos, vinaza, torta de filtro o cachaza y ceniza de caldera, entre otros, son reincorporados a la tierra convirtiéndose en abono natural orgánico.

Tabla 5

Características Físicas del Cultivo de Lechuga (Lactuca Sativa L.)

\begin{tabular}{ccccc}
\hline Tratamiento & $\begin{array}{c}\text { Longitud de } \\
\text { planta }(\mathrm{cm})\end{array}$ & $\begin{array}{c}\text { Diámetro } \\
\text { ecuatorial }(\mathrm{cm})\end{array}$ & $\begin{array}{c}\text { Peso de una } \\
\text { lechuga }(\mathrm{g} .)\end{array}$ & $\begin{array}{c}\text { Rendimiento } \\
(\mathrm{Tn} / \mathrm{ha})\end{array}$ \\
\hline $\mathrm{T}_{5}$ & $26.92 \mathrm{a}$ & $24.79 \mathrm{a}$ & $165.83 \mathrm{a}$ & $12.02 \mathrm{a}$ \\
$\mathrm{T}_{4}$ & $26.22 \mathrm{a} \mathrm{b}$ & $24.50 \mathrm{a}$ & $155.08 \mathrm{a}$ & $11.62 \mathrm{a}$ \\
$\mathrm{T}_{3}$ & $24.83 \mathrm{a} \mathrm{b}$ & $23.21 \mathrm{a} \mathrm{b}$ & $143.75 \mathrm{a}$ & $11.04 \mathrm{a}$ \\
$\mathrm{T}_{2}$ & $24.37 \mathrm{~b}$ & $22.92 \mathrm{a} \mathrm{b}$ & $129.38 \mathrm{a}$ & $9.93 \mathrm{a}$ \\
$\mathrm{T}_{1}$ & $24.08 \mathrm{~b}$ & $21.83 \mathrm{~b}$ & $119.58 \mathrm{a}$ & $9.26 \mathrm{a}$ \\
\hline Significación & $* *$ & ${ }^{* *}$ & ${ }^{* *}$ & ${ }^{* *}$ \\
CV: $\%$ & 5.00 & 5.26 & 16.47 & 20.18 \\
\hline
\end{tabular}

\begin{tabular}{cc}
\hline Tratamiento & $\begin{array}{c}\text { Longitud de } \\
\text { raí }(\mathrm{cm})\end{array}$ \\
\hline $\mathrm{T}_{4}$ & $9.000 \mathrm{a}$ \\
$\mathrm{T}_{5}$ & $8.041 \mathrm{ab}$ \\
$\mathrm{T}_{3}$ & $7.500 \mathrm{ab}$ \\
$\mathrm{T}_{2}$ & $7.333 \mathrm{ab}$ \\
$\mathrm{T}_{1}$ & $7.083 \mathrm{~b}$ \\
\hline Significación & $* *$ \\
CV: \% & 11.52 \\
\hline
\end{tabular}


EXPERIMENTO CON BIOL DE SUBPRODUCTOS DE AZÚCAR PARA MAYOR RENDIMIENTO ECOLÓGICO EN EL CULTIVO DE LECHUGA (LACTUCA SATIVA L.)

Tabla 6

Análisis Completo de Hojas, según las Dosis de Biol

\begin{tabular}{|c|c|c|c|c|c|c|}
\hline \multirow{2}{*}{$\begin{array}{l}\text { Porcentaje } \\
(\%)\end{array}$} & \multicolumn{5}{|c|}{ Tratamientos } & \multirow{2}{*}{$\begin{array}{c}\text { Valores } \\
\text { Normales }\end{array}$} \\
\hline & $\mathbf{T}_{1}$ & $T_{2}$ & $\mathbf{T}_{3}$ & $\mathbf{T}_{4}$ & $T_{5}$ & \\
\hline $\mathbf{N}$ & 3.94 & 3.59 & 3.45 & 3.24 & 3.66 & $\begin{array}{c}3.50- \\
6.00\end{array}$ \\
\hline $\mathbf{P}$ & 0.75 & 0.82 & 0.68 & 0.63 & 0.73 & $\begin{array}{c}0.40- \\
1.00\end{array}$ \\
\hline $\mathbf{K}$ & 4.16 & 4.24 & 3.58 & 3.60 & 3.26 & $\begin{array}{c}3.50- \\
8.00\end{array}$ \\
\hline $\mathrm{Ca}$ & 1.42 & 1.42 & 1.38 & 1.30 & 1.35 & $\begin{array}{l}1.25- \\
2.50\end{array}$ \\
\hline Mg & 0.68 & 0.69 & 0.61 & 0.60 & 0.61 & $\begin{array}{c}0.30- \\
1.00\end{array}$ \\
\hline $\mathrm{Na}$ & 0.41 & 0.44 & 0.38 & 0.51 & 0.37 & $\begin{array}{c}0.01- \\
0.20\end{array}$ \\
\hline \multicolumn{7}{|l|}{ Ppm } \\
\hline $\mathrm{Cu}$ & 2.3 & 2.1 & 2.0 & 2.1 & 2.3 & $6-20$ \\
\hline $\mathbf{F e}$ & 252.2 & 56.2 & 56.2 & 54.2 & 60.2 & $60-200$ \\
\hline $\mathbf{Z n}$ & 47.3 & 36.4 & 33.0 & 44.2 & 34.3 & $30-50$ \\
\hline B & 27.3 & 33.3 & 16.3 & 27.4 & 15.2 & $25-50$ \\
\hline
\end{tabular}

Adaptado de "Hoja de análisis foliar. Código 105 -108," por Gálvez, Legua, Cruz \& Caro, 2018. Instituto Nacional de Investigación Agraria - Huaral.

\subsection{Peso de una lechuga}

Este resultado se obtuvo del peso de las lechugas marcadas de cada tratamiento (16 lechugas). Tales cifras se muestran en la Tabla 5 y la Figura 2, con lo que se determinó el mayor peso en $\mathrm{T}_{5}$ con 165.83 g., siendo estadísticamente homogéneo al $\mathrm{T}_{4}$ con 155.08 g.; seguido del $\mathrm{T}_{3}$ con 143.75 g.; $\mathrm{T}_{2}$ con 129.38 g. y $\mathrm{T}_{1}$ con 119.58 g. en agrupamiento (a). Asimismo, se observa que no es significativo, lo que da a entender que no influenció la dosis de biol. De acuerdo con López, Andrade, Herrera, Gonzales y García (2015), el compost (base de subproductos de caña de azúcar) muestra indicadores positivos de calidad, cuantificándose la humedad de $59 \%$, el 
$\mathrm{pH}$ 8.2; la relación carbono-nitrógeno de 12.5; el nitrógeno de $1 \%$, fósforo $1.3 \%$, potasio $1.1 \%$ y magnesio $1.1 \%$, así como una baja salinidad. Siendo la aplicación de compost favorable en mayor dosis para la obtención de mayor peso de lechuga.

\subsection{Longitud total de planta}

En cuanto a los resultados de la longitud de la planta, se aprecia en la Tabla 5 que el $\mathrm{T}_{5}$ obtuvo $26.91 \mathrm{~cm}$, lo cual es estadísticamente homogéneo al $\mathrm{T}_{4}$ con $26.20 \mathrm{~cm}$, al $\mathrm{T}_{3}$ con $24.83 \mathrm{~cm}$ en grupo Duncan (a), diferente al $\mathrm{T}_{2}$ con 24.37 y $\mathrm{T}_{1}$ con $24.08 \mathrm{~cm}$ en Duncan agrupamiento (b). También se observa que no son significativos, es decir, no influencio la dosis de biol.

\subsection{Rendimiento comercial}

Los datos obtenidos con las dosis de biol determinaron que el $\mathrm{T}_{5}$ obtuvo el mayor rendimiento con $12.02 \mathrm{tn} / \mathrm{ha}$; siendo al $\mathrm{T}_{4}$ con $11.62 \mathrm{tn} / \mathrm{ha}$; $\mathrm{T}_{3}$ con $11.04 \mathrm{tn} / \mathrm{ha} ; \mathrm{T}_{2}$ con $9.93 \mathrm{tn} /$ ha y $\mathrm{T}_{1}$ con 9.26 todos estadísticamente homogéneos. Cabe mencionar que no hay significancia, por lo tanto, no influenció la concentración de biol.

\subsection{Diámetro ecuatorial}

Esta evaluación se realizó en el laboratorio. A partir de las plantas marcadas de cada tratamiento se midió con una regla el diámetro ecuatorial, resultado que se observa en la Tabla 5. El mayor diámetro se obtuvo en $\mathrm{T}_{5}$ con $24.79 \mathrm{~cm}$, siendo estadísticamente similar al $\mathrm{T}_{4}$ con $24.50 \mathrm{~cm}$ en Duncan agrupamiento (a); $\mathrm{T}_{3}$ con $23.20 \mathrm{~cm} \mathrm{y}_{2}$ con $22.91 \mathrm{~cm}$ en Duncan agrupamiento $(\mathrm{ab})$, diferenciándose de $\mathrm{T}_{1}$ con $21.83 \mathrm{~cm}$ en agrupamiento 
EXPERIMENTO CON BIOL DE SUBPRODUCTOS DE AZÚCAR PARA MAYOR RENDIMIENTO ECOLÓGICO EN EL CULTIVO DE LECHUGA (LACTUCA SATIVA L.)

(b). También se muestra que no es significativo, por lo que no influenció la dosis de biol.

\subsection{Longitud de raíz}

Después de le evaluación anterior, se obtuvo los resultados de la longitud de raíz. Esto se muestra en la Tabla 5, donde alcanzó mayor longitud en el $\mathrm{T}_{4}$ con $9.00 \mathrm{~cm}$, siendo estadísticamente homogéneo al $\mathrm{T}_{5}$ con $8.041 \mathrm{~cm} ; \mathrm{T}_{3}$ con $7.5 \mathrm{~cm} ; \mathrm{T}_{2}$ con $7.33 \mathrm{~cm}$ en Duncan agrupamiento (ab) y diferenciándose del $\mathrm{T}_{1}$ con 7.083. Duncan agrupamiento (b). Del mismo modo, se afirma que no es significativo, por lo que no influyó la concentración de biol.

\subsection{Análisis foliar de lechuga en porcentaje}

Los análisis obtenidos por el Instituto Nacional de Investigación Agraria - Huaral, mostrados en la Tabla 6, indican que disminuyen gradualmente los porcentajes de los macroelementos: nitrógeno, fósforo, potasio en el $\mathrm{T}_{4}$ con $750 \mathrm{~mL}$ y $\mathrm{T}_{5}$ con $1000 \mathrm{~mL}$ por $200 \mathrm{l}$ de agua, igual tendencia muestran los microelementos como calcio magnesio y sodio para los tratamientos $\mathrm{T}_{4} \mathrm{y}_{5}$, por lo tanto, se afirma que al aumentar la dosis de biol disminuye la concentración de los microelementos por 100 gr de materia seca, esta relación se ilustra en la Figura 5. Esta relación es favorable, pues aumentó el rendimiento comercial. Este resultado, se puede contrastar con Ospina (2016), quien afirma que una relación directa entre la dosis de compost aplicada y extracción de este nutriente, se asocia con el mayor aporte de poblaciones microbianas, que generan mayor descomposición de la materia orgánica y, a su vez, cambios en el proceso de óxido-reducción que permiten la disponibilidad de este nutriente. También, altas concentraciones 
de compost impactan positivamente en las variables agronómicas del cultivo de caña de azúcar, reflejándose en mayores contenidos de toneladas de caña por hectárea.

\subsection{Análisis foliar en partes por millón (ppm)}

Por análisis foliar se obtuvo los resultados de los microelementos, que se muestran en la Tabla 5 , que disminuyen en el $\mathrm{T}_{3}, \mathrm{~T}_{4}$ y $\mathrm{T}_{5}$ como fierro, zinc y boro. Sin embargo, el cobre se mantiene casi invariable en su concentración en todos los tratamientos en 2 ppm en promedio. También estos resultados son corroborados en la Figura 4, que muestra que a mayor dosis de biol disminuye la concentración de los microelementos, siendo esto beneficioso porque aumentó el rendimiento comercial. Esto se relaciona con Quintero (1995), mencionado por Ospina (2016), al afirmar que el cultivo de caña requiere cantidades bajas de los elementos menores para la función en la actividad fisiológica del Mn en este cultivo, lo cual está relacionada con la fotosíntesis y actividad enzimática.

\section{Conclusiones}

- Se obtuvo el mayor rendimiento con $12.02 \mathrm{tn} /$ ha de lechuga en el $\mathrm{T}_{5}$ con $1000 \mathrm{ml}$ de biol por $200 \mathrm{l}$. de agua diferenciándose de los demás tratamientos. A su vez, el menor rendimiento fue con 9.26 tn/ha en $T_{1}$ (testigo), lo cual establece que de esta concentración de aplicación se obtuvo mayor rendimiento comercial.

- De acuerdo a las características físicas de la lechuga, sobresalieron las mayores dosis: el $\mathrm{T}_{5}$ con $1000 \mathrm{ml}$ por $200 \mathrm{l}$, en longitud de planta obtuvo $26.92 \mathrm{~cm}$; en peso para una lechuga $165.83 \mathrm{~g} \mathrm{y}$ 
diámetro ecuatorial con $24.79 \mathrm{~cm}$. Sin embargo en $\mathrm{T}_{4}$ con $750 \mathrm{ml}$ por 2001 de agua, alcanzó mayor longitud de raíz con $9.00 \mathrm{~cm}$.

- Los análisis foliares obtenidos de cada tratamiento determinaron que a mayor dosis de biol disminuye la concentración de los elementos en el $\mathrm{T}_{4}$ con $750 \mathrm{ml} \mathrm{y}_{5}$ con $1000 \mathrm{ml}$ de biol por 200 1 de agua, siendo favorable porque aumentó el rendimiento de lechuga.

- También se determinó que la aplicación de biol obtuvo resultado no significativo, de lo que se interpreta que no influenció en el peso de una lechuga y rendimiento, pero se alcanzó a obtener un fruto ecológico.

\section{REFERENCIAS}

Bustamante, K. (2014). Análisis de la Situación Actual de las Emisiones del Ingenio Central Progreso, Veracruz. Trabajo Recepcional. Veracruz, México: Universidad Veracruzana. Recuperado de: https://cdigital.uv.mx/bitstream/handle/123456789/46736/ BustamanteMarinKaren.pdf?sequence=2\&isAllowed $=y$

Cando, S. \& Malca, L. (2015). Influencia de un abono orgánico líquido tipo biol en el rendimiento de la lechuga (Lactuca sativa L) cultivada en sistemas hidropónicos. Manglar. Revista de Investigación Cientifica, Universidad Nacional de Tumbes, 12(2), 31-38. 
Bello, A., García, A. \& Díez, M. (2004). Ecología de Suelos, Manejo de la Materia Orgánica e Investigación. I Conferencia Internacional EcoBiología del Suelo y el Compost. España: Edición SOILACE

Fondo de Cooperación para el Desarrollo Social. (2014). Producción y uso de abonos orgánicos: biol, compost y humus. Proyecto Mi Chacra Emprendedora - Haku Wiñay. Manual Técnico, N. 5. Lima, Perú: Ministerio de Desarrollo e Inclusión Social, Fondo de Cooperación para el Desarrollo Social.

Friedmann, A., Weil, B. \& Penner, R. (2010). Azúcar Orgánica Potencial de Negocios. Paraguay: Estados Unidos y para el Desarrollo Internacional (USAID)

Gálvez, E. Legua, J. Cruz, D. y Caro, F. (2018). Hoja de análisis de suelo. Código 105 -108. Instituto Nacional de Investigación Agraria Huaral.

Gálvez, E. Legua, J. Cruz, D. y Caro, F. (2018). Hoja de análisis foliar. Código 105 -108. Instituto Nacional de Investigación Agraria Huaral.

López, E. (2011). Metodología de la investigación: Guía Instruccional. Registro de publicación de la Universidad Nacional Abierta N. UNAGL-12-5971. Universidad Nacional Abierta. Caracas, Venezuela. Recuperado de https://docplayer.es/9472077-Metodologia-de-lainvestigacion.html

López, E., Andrade, A., Herrera, S., Gonzalez, O. \& García, A. (2015). Propiedades de un compost obtenido a partir de residuos de la producción de azúcar de caña. Revista Centro Agrícola, 44(3), 


\section{EXPERIMENTO CON BIOL DE SUBPRODUCTOS DE AZÚCAR PARA MAYOR RENDIMIENTO}

ECOLÓGICO EN EL CULTIVO DE LECHUGA (LACTUCA SATIVA L.)

49-55. Recuperado de http://scielo.sld.cu/scielo.php?script=sci_ arttext\&pid=S0253-57852017000300007

López, E., \& González, B. (2014). Diseño y Análisis de Experimentos

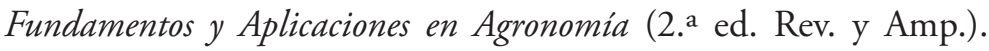
Guatemala. Recuperado de https://docplayer.es/49683224-Diseno$\mathrm{y}$-analisis-de-experimentos.html

Núñez, V., \& Tusell, F. (2007). Regresión y Análisis de Varianza. España. Recuperado de http:/www.et.bs.ehu.es/ - etptupaf/nuevo/ficheros/ estad3/reg.pdf

Organización de las Naciones Unidas para la Alimentación y la Agricultura. (2002). Los fertilizantes y su uso. Roma, Italia: Organización de las Naciones Unidas para la Alimentación y la Agricultura, Asociación Internacional de los Fertilizantes. Recuperado de: http://www. fao.org/documents/card/es/c/b0 f8bfc5-4c95-54b0-80cd96b810006037/

Ospina, I. (2016). Influencia de la aplicación de compost producido a partir de residuos de la caña de azúcar (Saccharum officinarum L.) en un Vertisol de Valle del Cauca (Tesis presentada como requisito parcial para optar al título de Magíster en Ciencia Agrarias, Universidad Nacional de Colombia. Palmira, Colombia).

Quintero, R. (1995). Fertilización y nutrición. En Cenicaña, El cultivo de la caña en la zona azucarera de Colombia. Cali, Colombia: Cenicaña.

Quiroga, A. \& Bono, A. (2012). Manual de fertilidad y evaluación de suelo. Proyecto Regional Mixto. Área Estratégica de Gestión del Agua y Área Estratégica de Recursos Naturales. Instituto Nacional de Tecnología Agropecuaria. Argentina. Recuperado de https:// 
EDWIN GÁLVEZ TORRES, JOSÉ LEGUA CÁRDENAS, DANTE DANIEL CRUZ NIETO Y FÉLIX CARO SOTO

inta.gob.ar/sites/default/files/script-tmp-inta_pt_89_manual_de_ fertilidad_1_____.pdf 